\title{
FDG-PET-CT in the early response evaluation for primary systemic therapy of breast cancer
}

\section{Case Report}

Tímea Tókés*1, László Torgyík ${ }^{1}$, Janina Kulka², Katalin Borka², Attila Marcell Szász², Andrea Tóth ${ }^{1}$, László Harsányi ${ }^{3}$, Zsolt Lengyel ${ }^{4}$, Tamás Györke ${ }^{5,6}$, Magdolna Dank ${ }^{1}$

$11^{\text {st }}$ Department of Internal Medicine, Oncology Division Semmelweis University, Hungary, Budapest, 1083, Tömö u. 25-29

$22^{\text {nd }}$ Department of Pathology, Semmelweis University, Hungary, Budapest, 1091, Üllói út 93

$31^{\text {st }}$ Department of Surgery, Semmelweis University, Hungary, Budapest, 1082, Üllói út 78

4 Pozitron Diagnostics Ltd. Budapest, Hungary, Budapest, 1117, Hunyadi J. út 9

5 Department of Nuclear Medicine, Semmelweis University Hungary, Budapest, 1082, Üllői út 78/A

6 Scanomed Ltd. Budapest, Hungary, Budapest, 1145, Laky Adolf u. 44-46.

\section{Received 24 April 2013; Accepted 6 August 2013}

Abstract: Primary systemic therapy (PST) is a standard treatment for patients with locally advanced breast cancer. We report one of our patients to demonstrate the optimal use of FDG-PET-CT in the routine clinical workup during PST, especially when clinicians face contradictory clinical and pathological findings, and to show the advantages of this imaging modality in the decision-making process about the initial treatment choice. By reviewing the literature we would also like to confirm that FDG-PET-CT is highly sensitive in the measurement of the early therapeutic response and the prediction of the complete pathological remission, as early as after the first cycle of chemotherapy is administered.

Keywords: Breast cancer • Primary systemic therapy • FDG-PET-CT •Interim • Ki-67

(C) Versita Sp. z 0.0

\section{Introduction}

Primary systemic therapy (PST) has become a standard treatment for patients with locally advanced breast cancer and inflammatory breast cancer. The first goal of PST is to assess the sensitivity of the tumor for chemotherapy, namely that PST is an "in vivo test" for the drug resistance. The treatment can be optimized, and PST may allow performance of breast-conserving surgery, with down-staging of the tumors [1-3]. There are many contradictions in the literature about primary systemic therapy, but many studies have shown that with PST - particularly when complete pathological remission (same as pathologic complete response $=$ pCR) was achieved - the overall survival (OS), the disease-free survival (DFS) and relapse-free survival (RFS) were significantly longer [1,4-8].

To measure the response to the therapy is essential, and to clearly identify the responder and non-responder

*E-mail: tokes.timea@med.semmelweis-univ.hu; timi.tokes@gmail.com 
patients is crucial [9]. During the morphological response evaluation, even by using increasingly sensitive imaging modalities, it was found that during cancer treatment the metabolic changes were earlier in onset than the morphological response. This has led to the era of metabolic imaging - especially positron emission tomography (PET) - in tumor response evaluation [8]. The PET-CT imaging - using [18F]-fluoro-deoxy-glucose(FDG) - is based on the increased metabolism and glucose uptake of tumors. Its biggest advantage over PET alone is the fusion of functional and anatomical imaging - owing to this it can be highly sensitive in the localization of viable tumor tissue in the body. Moreover FDG-PET can identify the therapy-induced changes in tumor metabolism with high sensitivity. Using FDG-PET-CT it was already found that after 1 or 2 cycles of anticancer therapy (interim PET-CT imaging) the responder and non-responder patients could be separated effectively $[10,11]$. With the detection of the metabolic response in an early phase, we could acquire information on the effectiveness of the systemic therapy well in advance compared to the morphological methods.

In our country interim PET for breast cancer could only be performed if the tumor showed equivocal clinical behavior and the result of the interim PET-CT may lead to changes in the chemotherapy regimen to be administered. Otherwise only presurgical - after 4 or 6 cycles of PST - PET-CT is used to evaluate the therapeutic response for neoadjuvant treatment. In our case study we report one of our patients to demonstrate that if pathological findings and clinical behavior are not consistent, then the interim PET-CT shall decide if the clinician selected the right therapy to gain pathologic complete response ( $p C R)$.

\section{Case Report}

\subsection{Patient history}

Our 52-year old patient had discovered a lump in her right breast by self-examination. One and a half years before her first "detection" she had participated in routine mammography screening with negative results. She was presented in our unit with 4-6 week history of a rapidly growing lump in her right breast. The patient had no history of contraceptive medication use and she has never been pregnant $(0 / 0)$. She was postmenopausal due to gynecological surgery for endometriosis, which included hysterectomy and bilateral adnexectomy (at the age of 47). Following this operation she was put on hormone-replacement therapy due to her symptoms for 3 months.

\subsection{Tumor presentation}

On physical examination, the medical oncologist palpated a lesion with nearly $4 \mathrm{~cm}$ in diameter in the right breast, at the border of the upper-outer quadrant. The skin of the breast showed mild signs of inflammation. Unfortunately, the patient did not consent to photo documentation of the symptoms.

During diagnostic imaging workup, the mammography detected a spiculated lesion with $2.5 \mathrm{~cm}$ longest diameter in the upper-outer quadrant. The breast ultrasound revealed a $27 \times 20 \mathrm{~mm}$ hypoechogenic nonhomogeneous mass in the upper outer quadrant of the right breast along with an enlarged lymph node in the axilla. Ultrasound guided fine needle aspiration biopsy (FNAB) was performed from the breast lesion. The result showed unequivocal malignancy with invasive grade 3 breast cancer cells (C5).

\subsection{Treatment planning}

The multidisciplinary team of our department decided to administer PST because of the rapid growth of the tumor and its mastitis-like behavior. Before initiating treatment, ultrasound guided histological sampling (core-biopsy) was taken and PET-CT staging was performed.

The core-biopsy indicated nuclear grade 3 invasive ductal carcinoma with moderate mitotic activity (9 mitoses/ 10 high power fields). The immuno-histochemistry test provided the following results:

- estrogen, progesterone receptors and HER2: negative;

- Ki-67 proliferation marker: $10 \%$, p53 tumor suppressor expression: $100 \%$;

- E-cadherin: positive, CK-7: positive, CK 5/6 negative.

Based on these results the cancer was classified as a high grade invasive ductal carcinoma (IDC) with triplenegative characteristics. Interestingly the proliferation index was rather low despite the rapid growth of the tumor according to the clinical history.

\subsection{FDG-PET-CT staging}

The PET scan was performed using GE Discovery ${ }^{\mathrm{TM}} \mathrm{ST}$ 8 PET-CT device (GE Medical Systems). Standardized Uptake Values (SUV) were calculated and morphological data were measured on staging PET-CT images for the primary tumor and axillary lymph node region. To measure the therapeutic response the maximum value of SUV was calculated (SUV ${ }_{\max }$ ) in each region (Table 1).

In the upper-outer quadrant of the right breast FDGPET-CT showed a metabolically active tumor; also the 
skin showed mild metabolic activity in the region of the clinical symptoms, i.e. the erythema. Furthermore, a metastatic lymph node with pathologic FDG-uptake in the right axillary region was detected. There was no sign of distant metastasis, therefore clinical TNM stage was established as cT2cN1M0.

\subsection{Primary systemic therapy}

The multidisciplinary team decided to administer PST. The patient received 6 cycles of taxane-platina based PST (docetaxel-carboplatin). After the first administered cycle of the PST, the erythema and the mastitis-like symptoms were resolved almost completely, while the palpable lesion did not shrink significantly. The treating physician decided to order an interim PET-CT, to find out whether the metabolism of the tumor decreased in line with the symptoms of the skin.
The interim PET-CT scan revealed metabolic remission, which was more obvious than the morphological one. In the axillary lymph node region the metabolism also decreased markedly, and the morphological response was better than that in the primary tumor region, according to RECIST 1.1 [12] complete morphological remission was detected (Table 1 ).

At the end of the sixth cycle, before the surgery, a restaging PET-CT was performed. There was no sign of metabolic activity in the primary tumor, in contrast to the morphological change that is the total shrinkage reached only 27.5 percent reduction in the longest diameter of the primary tumor, which meant - according to RECIST 1.1 - a stable disease. The metabolically active axillary lymph node showed moderate uptake on the restaging PET-CT and its size did not reduce any further in the short axis diameter since the interim PET-CT scan (Table 1).

Table 1. FDG-PET-CT results

\begin{tabular}{l|llll}
\hline PET-CT scans & Primary Tumor SUV & Primary Tumor Size & Axilla SUV & Axilla Size \\
\hline \hline Staging PET & 15,7 & $32 \times 40$ & 17,7 & $17 \times 22$ \\
Interim PET & 5,3 & $24 \times 30$ & 4,0 & $10 \times 16$ \\
Restaging PET & 1,7 & $19 \times 29$ & 2,5 & $10 \times 12$ \\
Interim & $\Delta$ SUV $=10,4$ & $25 \%$ & $\Delta S U V=13,7$ & $41 \%$ \\
response & $66,3 \%$ & RECIST: SD & $77,4 \%$ & RECIST: CR \\
Overall response & $\Delta$ SUV $=14$ & $27,5 \%$ & $\Delta S U V=15,2$ & $41 \%$ \\
& $89,2 \%$ & RECIST: SD & $85,88 \%$ & RECIST: CR \\
\hline
\end{tabular}

[SUV: Standardized Uptake Value; RECIST: Response Evaluation Criteria In Solid Tumors (version used: 1.1), SD: stable disease, CR: complete remission

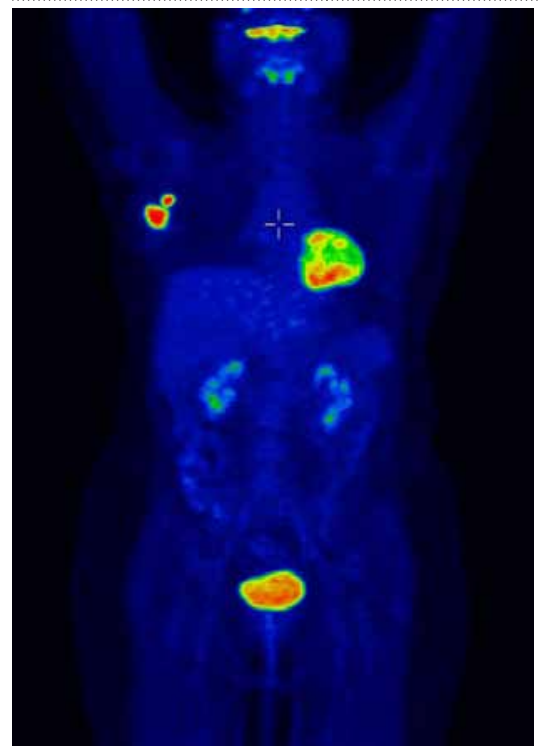

Figure 1A

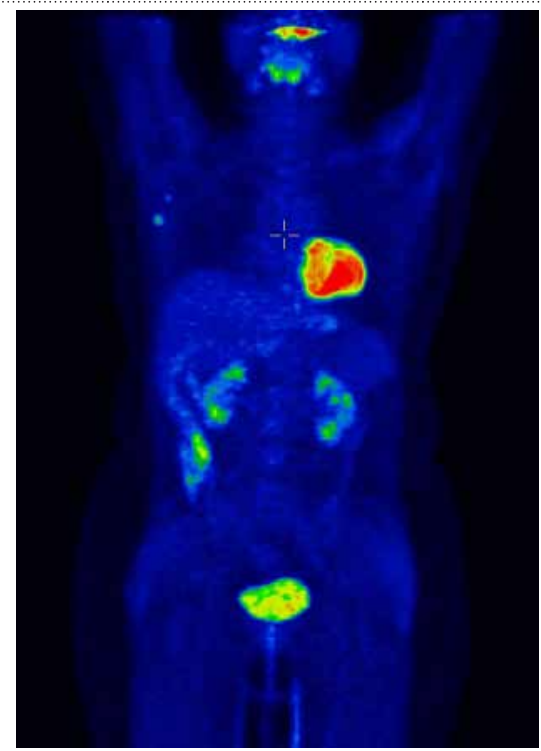

Figure 1B

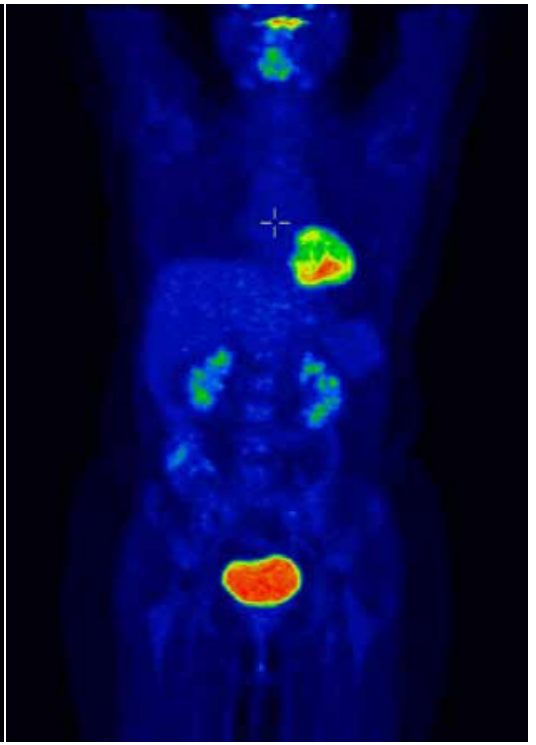

Figure 1C

Figure 1 A-C. Staging (Fig.1A), interim (Fig.1B) and restaging (Fig.1C) FDG-PET-CT investigations; MIP (Maximum Intensity Projection) PET images. On the staging image (Fig.1C) the metabolically active tumor in the right breast and one metastatic lymph node were visible (arrow). The interim images (after C01) showed metabolic regression in both regions (Fig.1B), and on the restaging scans (after $\mathrm{C} 06$ ) the primary tumor showed metabolic complete remission, with slight residual uptake in the axillary region (Fig. 1C). 


\subsection{Surgery and adjuvant therapy}

In the surgical department, breast conserving surgery (sector excision) with axillary block dissection was performed. The final histological examination of the surgical specimen provided the following results:

- The sample of the sector excision showed pathologic complete response in the breast, that is there was no sign of viable tumor cells in the specimen; just necrotic and fibrotic breast tissue was found (pCR).

- In the axillary block specimen - which contained 10 lymph nodes - two nodes were infiltrated by tumor cells. The immuno-histochemistry resulted in a triple negative IDC with $80 \%$ of Ki-67 labeling index and $100 \%$ of p53 staining.

- The pathological response rate was established as Sataloff T-A, N-D.

In conclusion, according to the pathological staging, the patient was in ypTOypN1a (2/10) TNM stage, and according to the PET-CT scan performed before surgery - clinically free of distant metastases.

After surgery the patient received adjuvant chemotherapy because of the high proliferation rate detected in the axillary lymph node metastases, followed by radiation therapy (50-50/2 Gray for the right breast- and axillary-region).

At the time of composition of this paper the patient had been in complete remission. The last treatment was administered almost 2 years ago; 32 months elapsed since the diagnosis was made.

\section{Discussion}

FDG-PET-CT is able to measure changes in glucose metabolism induced by the therapy in the tumor tissue with high precision. This makes it a distinguished tool for monitoring therapy [13]. Most of the authors found an excellent correlation between the tumors's FDG-uptake and the response of the tumor after the first cycle of chemotherapy. In the paper of Wahl et al. [14], the reduction in FDG-uptake during the evaluation of the therapeutic response with PET-CT after the first cycle of PST (on the 8th day from the beginning of the therapy) was predictive for complete pathological remission. Lee et al. described (summarizing 9 different studies) that after the first or second cycle of chemotherapy, early assessment of response with FDG-PET was predictive for the final pathological response [15]. Wang et al. revealed in their meta-analysis (16 studies) that $55-65 \%$ cut off reduction of the SUV after the 1st or 2nd cycle has the best correlation with pathological response [16]. In the following table (Table 2) we give a summary of those studies that used PET-CT after the first cycle of PST and defined cut-off values for the prediction of the pCR.

Rousseau et al. analyzed the sensitivity, the specificity and the negative predictive value of FDG-PET-CT with $40 \%$ decrease in the SUV as the threshold for response. The sensitivity, the specificity and the negative predictive values were $61 \%, 96 \%$ and $68 \%$ after the first cycle, 89-95-85\% after the second cycle and 88$73-83 \%$ after third cycle for the pathological response, respectively [17]. In summary, they suggested that the best time for the assessment of the metabolic response is after the second cycle of the neoadjuvant therapy. Groheux et al. - through analyzing 9 studies - suggested the same optimal timing for the interim PET-CT [23]. Berriolo-Riedinger et al. established in their study [18] - response evaluation in 47 patients after the first cycle - that, when using $60 \% \Delta S U V$ cut-off values, the FDG-PET-CTs were highly predictive for the pathologic complete response ( $p C R)$. Schwarz-Dose et al. came to the conclusion that, after the first and the second course,

Table 2. Studies evaluating the metabolic response with FDG-PET-CT after the first cycle of PST.

\begin{tabular}{|c|c|c|}
\hline Study & Timing of interim PET & PET criteria* \\
\hline Schelling et al.[10] & after 1 st, 2nd cycle & $-55 \%$ (after 2nd cycle) \\
\hline Smith et al. [11] & $\begin{array}{l}\text { after } 1 \text { st, } 2 \text { nd, } 5 \text { th cycle } \\
\text { dynamic PET-CT performed }\end{array}$ & DUR $^{* *}>20 \%$ (after 1 st cycle) \\
\hline Rousseau et al.[17] & after 1st, 2nd, 3rd course & $-40 \%$ (after 2nd cycle) \\
\hline Berriolo-Riedinger et al.[18] & after 1 st cycle & $-60 \%$ (after 1st cycle) \\
\hline Ueda et al.[19] & after 1 st cycle & $-40 \%$ after 1 st cycle \\
\hline Schwarz-Dose et al.[20] & after 1 st and 2 nd cycle & $\begin{array}{l}-45 \% \text { after } 1 \text { st cycle } \\
-55 \% \text { after } 2 \text { nd cycle }\end{array}$ \\
\hline Keam et al. [21] & after 1 st cycle & $-50 \%$ after 1 st cycle \\
\hline Kolesnikov-Gauthier et al.[22] & after 1st cycle & decrease less than $15 \%$ is predictive for therapy failure \\
\hline
\end{tabular}

*decrease in the percent of the baseline (pretherapy) SUV

${ }^{*}$ DUR $=$ dose uptake ratio (Patlak analysis, FDG-uptake calculated by voxels on the dynamic and static scans in Smith et al. study) 
PET performed equally. [20]. Kolesnikov-Gauthier et al. revealed, that less than $15 \%$ decrease in the SUV after the first cycle of the chemotherapy is highly predictive for therapy failure [22]. In the case of our patient we found more than $60 \%$ decrease in the SUV, and later the histology confirmed the pCR, so the interim PET-CT results were predictive of the pathological remission.

The FDG-uptake depends on several biological properties of the tumors. The highest level of correlation was found with the glucose metabolism of the cell (e.g. GLUT-1 expression) and with the Ki-67 proliferation marker [24,25]. It is well documented that using Ki-67 labeling index (LI) the response to therapy is predictable - the high number of mitotic cells suggests good response for cytotoxic treatment. Furthermore Ki-67 LI plays an important role in the prognostic assessment of the tumor as well [26-28]. The correlation between Ki-67 $\mathrm{LI}$ and the FDG-uptake of the tumor is a frequently examined question. Buck et al. showed that low proliferation activity was associated with low FDG-uptake, and inversely, high Ki-67 expression was associated with high FDG-avidity [25]. Buck [24], Avril [29], and Bos [30] confirmed that in invasive ductal carcinomas the Ki-67 $\mathrm{LI}$ is higher than in the lobular type tumors. Shimoda et al. [31], Gil-Rendo et al. [32] and Koolen et al. [33] found significant correlation between $\mathrm{Ki}-67 \mathrm{LI}$ and the SUV $_{\text {max }}$, as well as between the mitotic count and the FDG-uptake. Tchou et al. reported that triple negative breast cancer displays higher Ki-67 expression, grade and mitotic count than other histological subtypes and their FDG-uptake is also significantly higher [34].

In our case we found apparently conflicting results. The core-biopsy showed low Ki-67 LI, although the other pathological properties suggested aggressive biological behavior of the tumor and the mitotic activity was also moderate. The aggressive behavior and the relatively low proliferation index suggested different chances of the outcome to the neoadjuvant treatment. On the contrary, in the surgical specimen of the involved axillary lymph nodes the Ki-67 index was high (80\%) and complete pathological remission was observed in the primary tumor - that maybe suggests high proliferation rate before the therapy.

The possible causes of these contradictions are as follows: the measurement of $\mathrm{Ki}-67 \mathrm{LI}$ is cheap and easy to perform in every pathological department, but it has shortfalls related to the subjective judgment of the pathologist, the intra- and inter-observer variability (especially in Grade 2 carcinomas), and the differences between the applied antibodies [28,35]. Furthermore, intra-tumor variations of MIB-1 scoring were also reported in the assessment of individual cancers by lqbal et al [36], which suggest that the place of the sampling and the number of the specimens are important factors in case of core-biopsies as well. But in the measurement of the cell proliferation, the Ki-67 expression is the only validated and trusted marker nowadays in spite of its disadvantages in the therapeutic decision for breast cancer management [37].

By knowing these limitations of the immunohistochemistry, our multidisciplinary team decided to administer PST, based on the clinical data, the mastitislike symptoms, the rapidly growing lesion and the high metabolic activity of the tumor, with strict monitoring of the tumor response, because of the relatively low $\mathrm{Ki}-67 \mathrm{LI}$ in the core-biopsy. After considering the results of the interim and pre-surgical PET-CT scans and the pathological examination of the surgical specimen, we suspect that the core-biopsy sampling was not fully representative for some of the biological properties of the tumor (although the different proliferation rate in the axillary region could be caused by changes in the biological behavior during the metastatic spreading in the lymphoid system, as well). This is the underlying advantage of FDG-PET-CT: it maps the whole tumor, not just a representative part of the lesion. Moreover the whole-body scan also gives information about the locoregional and distant spread of the tumor.

\section{Conclusions}

The FDG-PET-CT is highly recommended for staging and it has clear advantages in therapy monitoring. Metabolic changes detected by FDG-PET are sensitive markers for therapeutic response, especially in predicting the pathological remission. This case was really instructive for the indication during the PST: if some of the core-biopsy results and the clinical behavior are not consistent, the FDG-PET-CT results could aid in clinical decision-making. With the routine use of interim imaging techniques during neoadjuvant therapy, the ineffective treatments could safely be modified and the effective therapy could be prolonged or enhanced in an earlier phase of the treatment. This new perspective in the management of primary systemic therapy might offer an early opportunity for individualized cancer treatment, which is our main goal in daily practice.

\section{Conflict of interest}

The authors have no conflict of interest to declare 


\section{References}

[1] Kaufmann M, von Minckwitz G, Smith R, Valero V, Gianni L, Eiermann W et al. International expert panel on the use of primary (preoperative) systemic treatment of operable breast cancer: review and recommendations. J Clin Oncol, 2003, 21, 2600-2608

[2] Kaufmann M, Hortobagyi GN, Goldhirsch A, Scholl $S$, Makris A, Valagussa $P$ et al. Recommendations from an international expert panel on the use of neoadjuvant (primary) systemic treatment of operable breast cancer: an update. J Clin Oncol, 2006, 24, 1940-1949

[3] Bonadonna G, Veronesi U, Brambilla C, Ferrari L, Luini A, Greco M et al. Primary chemotherapy to avoid mastectomy in tumors with diameters of three centimeters or more. J Natl Cancer Inst, 1990, 82, 1539-1545

[4] Kulka J, Tokes AM, Toth AI, Szasz AM, Farkas A, Borka $\mathrm{K}$ et al. [Immunohistochemical phenotype of breast carcinomas predicts the effectiveness of primary systemic therapy]. Magy Onkol, 2009, 53, 335-343

[5] Fisher B, Bryant J, Wolmark N, Mamounas E, Brown A, Fisher ER et al. Effect of preoperative chemotherapy on the outcome of women with operable breast cancer. J Clin Oncol, 1998, 16, 2672-2685

[6] Fisher B, Brown A, Mamounas E, Wieand S, Robidoux A, Margolese RG et al. Effect of preoperative chemotherapy on local-regional disease in women with operable breast cancer: findings from National Surgical Adjuvant Breast and Bowel Project B-18. J Clin Oncol, 1997, 15, 2483-2493

[7] Wolff AC, Davidson NE. Preoperative therapy in breast cancer: lessons from the treatment of locally advanced disease. Oncologist, 2002, 7, 239-245

[8] Sachelarie I, Grossbard ML, Chadha M, Feldman $\mathrm{S}$, Ghesani M, Blum RH. Primary systemic therapy of breast cancer. Oncologist, 2006, 11, 574-589

[9] Tardivon AA, Ollivier L, El Khoury C, Thibault F. Monitoring therapeutic efficacy in breast carcinomas. Eur Radiol, 2006, 16, 2549-2558

[10] Schelling M, Avril N, Nahrig J, Kuhn W, Romer W, Sattler $\mathrm{D}$ et al. Positron emission tomography using [(18)F]Fluorodeoxyglucose for monitoring primary chemotherapy in breast cancer. J Clin Oncol, 2000, 18, 1689-1695

[11] Smith IC, Welch AE, Hutcheon AW, Miller ID, Payne $\mathrm{S}$, Chilcott $\mathrm{F}$ et al. Positron emission tomography using [(18)F]-fluorodeoxy-D-glucose to predict the pathologic response of breast cancer to primary chemotherapy. J Clin Oncol, 2000, 18, 1676-1688

[12] Eisenhauer EA, Therasse P, Bogaerts J, Schwartz LH, Sargent D, Ford R et al. New response evaluation criteria in solid tumours: revised RECIST guideline (version 1.1). Eur J Cancer, 2009, 45, 228-247

[13] Forrai G, Szabo E, Ormandi K, Ambrozay E Pentek Z, Milics $\mathrm{M}$ et al. [Imaging methods in the current diagnosis of and screening for breast cancer]. Magy Onkol, 2010, 54, 211-216

[14] Wahl RL, Zasadny K, Helvie M, Hutchins GD, Weber B, Cody R. Metabolic monitoring of breast cancer chemohormonotherapy using positron emission tomography: initial evaluation. J Clin Oncol, 1993, 11, 2101-2111

[15] Lee JH, Rosen EL, Mankoff DA. The role of radiotracer imaging in the diagnosis and management of patients with breast cancer: part 2-response to therapy, other indications, and future directions. J Nucl Med, 2009, 50, 738-748

[16] Wang Y, Zhang C, Liu J, Huang G. Is 18F-FDG PET accurate to predict neoadjuvant therapy response in breast cancer? A meta-analysis. Breast Cancer Res Treat, 2012, 131, 357-369

[17] Rousseau C, Devillers A, Sagan C, Ferrer L, Bridji $B$, Campion $L$ et al. Monitoring of early response to neoadjuvant chemotherapy in stage II and III breast cancer by [18F]fluorodeoxyglucose positron emission tomography. J Clin Oncol, 2006, 24, 5366-5372

[18] Berriolo-Riedinger A, Touzery C, Riedinger JM Toubeau M, Coudert B, Arnould L et al. [18F]FDGPET predicts complete pathological response of breast cancer to neoadjuvant chemotherapy. Eur J Nucl Med Mol Imaging, 2007, 34, 1915-1924

[19] Ueda S, Tsuda H, Saeki T, Omata J, Osaki A, Shigekawa $\mathrm{T}$ et al. Early metabolic response to neoadjuvant letrozole, measured by FDG PET/CT, is correlated with a decrease in the Ki67 labeling index in patients with hormone receptor-positive primary breast cancer: a pilot study. Breast Cancer, 2011, 18, 299-308

[20] Schwarz-Dose J, Untch M, Tiling R, Sassen S, Mahner S, Kahlert S et al. Monitoring primary systemic therapy of large and locally advanced breast cancer by using sequential positron emission tomography imaging with [18F]fluorodeoxyglucose. J Clin Oncol , 2009, 27, 535-541 
[21] Keam B, Im SA, Koh Y, Han SW, Oh DY, Cho N et al. Early metabolic response using FDG PET/CT and molecular phenotypes of breast cancer treated with neoadjuvant chemotherapy. BMC cancer, 2011, 11, 452

[22] Kolesnikov-Gauthier H, Vanlemmens L, Baranzelli MC, Vennin P, Servent V, Fournier $C$ et al. Predictive value of neoadjuvant chemotherapy failure in breast cancer using FDG-PET after the first course. Breast Cancer Res Treat, 2012, 131, 517-525

[23] Groheux D, Giacchetti S, Espie M, Rubello D, Moretti JL, Hindie E. Early monitoring of response to neoadjuvant chemotherapy in breast cancer with 18F-FDG PET/CT: defining a clinical aim. Eur J Nucl Med Mol Imaging, 2011, 38, 419-425

[24] Buck A, Schirrmeister H, Kuhn T, Shen C, Kalker $\mathrm{T}$, Kotzerke $\mathrm{J}$ et al. FDG uptake in breast cancer: correlation with biological and clinical prognostic parameters. Eur J Nucl Med Mol Imaging, 2002, 29, 1317-1323

[25] Buck AK, Schirrmeister H, Mattfeldt T, Reske SN. Biological characterisation of breast cancer by means of PET. Eur J Nucl Med Mol Imaging 2004, 31 Suppl 1, S80-87

[26] Buban T, Toth L, Tanyi M, Kappelmayer J, AntalSzalmas P. [Ki-67 - new faces of an old player]. Orv Hetil, 2009, 150, 1059-1070

[27] de Azambuja E, Cardoso F, de Castro G, Jr., Colozza M, Mano MS, Durbecq $\mathrm{V}$ et al. Ki-67 as prognostic marker in early breast cancer: a metaanalysis of published studies involving 12,155 patients. Br J Cancer, 2007, 96, 1504-1513

[28] Colozza M, Azambuja E, Cardoso F, Sotiriou C, Larsimont D, Piccart MJ. Proliferative markers as prognostic and predictive tools in early breast cancer: where are we now? Ann Oncol, 2005, 16, 1723-1739

[29] Avril N, Menzel M, Dose J, Schelling M, Weber W, Janicke $F$ et al. Glucose metabolism of breast cancer assessed by 18F-FDG PET: histologic and immunohistochemical tissue analysis. J Nucl Med, 2001, 42, 9-16
[30] Bos R, van Der Hoeven JJ, van Der Wall E, van Der Groep P, van Diest PJ, Comans EF et al. Biologic correlates of (18)fluorodeoxyglucose uptake in human breast cancer measured by positron emission tomography. J Clin Oncol, 2002, 20, 379-387

[31] Shimoda W, Hayashi M, Murakami K, Oyama T, Sunagawa M. The relationship between FDG uptake in PET scans and biological behavior in breast cancer. Breast Cancer, 2007, 14, 260-268

[32] Gil-Rendo A, Martinez-Regueira F, Zornoza G, Garcia-Velloso MJ, Beorlegui C, Rodriguez-Spiteri N. Association between [18F]fluorodeoxyglucose uptake and prognostic parameters in breast cancer. Br J Surg, 2009, 96, 166-170

[33] Koolen BB, Vrancken Peeters MJ, Wesseling J, Lips EH, Vogel WV, Aukema TS et al. Association of primary tumour FDG uptake with clinical, histopathological and molecular characteristics in breast cancer patients scheduled for neoadjuvant chemotherapy. Eur J Nucl Med Mol Imaging, 2012, 39, 1830-1838

[34] Tchou J, Sonnad SS, Bergey MR, Basu S, Tomaszewski J, Alavi A et al. Degree of tumor FDG uptake correlates with proliferation index in triple negative breast cancer. Mol Imaging Biol, 2010, 12, 657-662

[35] Varga Z, Diebold J, Dommann-Scherrer C, Frick $H$, Kaup D, Noske A et al. How reliable is Ki-67 immunohistochemistry in grade 2 breast carcinomas? A QA study of the Swiss Working Group of Breast- and Gynecopathologists. PloS one, 2012, 7, e37379

[36] Iqbal S, Anderson TJ, Marson LP, Prescott RJ, Dixon JM, Miller WR. MIB-1 assessments in breast cancers. Breast, 2002, 11, 252-256

[37] Berruti A, Generali D, Kaufmann M, Puztai L, Curigliano G, Aglietta M et al. International expert consensus on primary systemic therapy in the management of early breast cancer: highlights of the Fourth Symposium on Primary Systemic Therapy in the Management of Operable Breast Cancer, Cremona, Italy (2010). Journal of the National Cancer Institute Monographs, 2011, 147-151 